\title{
MENINGITIS INFECCIOSA: PERFIL EPIDEMIOLÓGICO Y COMPORTAMIENTO CLÍNICO HOSPITAL CENTRAL DE LA POLICÍA, BOGOTÁ DC, COLOMBIA. 2004-2010
}

\author{
Jean Paul Vergara MD*, Wilson Puentes Cárdenas MD**
}

\begin{abstract}
Resumen
La meningitis es la infección más común del sistema nervioso central y el grupo estudiado es importante por tener factores de riesgo diferentes a la población general. Objetivo: describir el comportamiento clínico, diagnóstico, tratamiento y hallazgos paraclínicos en la población con meningitis infecciosa del Hospital Central de La Policía de Bogotá DC, durante el período 2004-2010. Materiales y métodos: estudio observacional descriptivo tipo serie de casos. Se analizaron las variables cualitativas en forma de frecuencia absolutas y relativas, las cuantitativas mediante medidas de tendencia central y de dispersión. Se utilizó Pasw Statistics 18 (licencia Universidad el Bosque). Resultados: se estudiaron 42 pacientes con promedio de 30,44 años; $30(71,4 \%)$ tenían comorbilidades, de ellos 25 no las tenían agudas mientras 17 pacientes $(40,5 \%)$ sí. El LCR fue turbio en 31 casos $(\mathbf{7 3 , 8 \%})$, se practicó gram de LCR en 30 muestras y fue positivo en $14(33,3 \%)$. Siete de los 42 pacientes murieron durante el tiempo en el que padecieron meningitis (16,7\%).
\end{abstract}

Palabras clave: meningitis, líquido cefaloraquídeo (LCR), comorbilidad, mortalidad, signos meníngeos.

Abreviaturas: SNC, sistema nervioso central; MB, meningitis bacteriana; LCR, líquido cefalorraquídeo.

\section{BACTERIAL MENINGITIS: EPIDEMIOLOGICAL PROFILE AND CLINICAL BEHAVIOR}

\begin{abstract}
Bacterial meningitis is the most common infection of the central nervous system. This study group is important for featuring risk factors different to those of the general population. Objective: to describe clinical behavior, diagnosis, treatment and lab testing findings in cases of bacterial meningitis occurring at Hospital Central de La Policía, Bogotá DC, during 2004 to 2010. Material and Methods: case series, descriptive, observational study. Qualitative variables were analyzed using absolute and relative frequencies. The quantitative variables were determined by measures of central tendency and dispersion. The Pasw Statistics 18 system (licensed by Universidad el Bosque) was used. Results: the study included 42 patients, mean age: 30.44 years; 30 (71.4\%) had comorbidities, 25 were not acute, while 17 (40.5\%) were. Cloudy CSF was found in 31 cases (73.8\%), Gram stain was performed in 30 samples of CSF and was positive in $14(33.3 \%)$. Seven of the 42 patients died during the period they presented meningitis $(16.7 \%)$.
\end{abstract}

Key words: meningitis, cerebrospinal fluid (CSF), comorbidity, mortality, meningeal signs

Fecha recibido: mayo 5 de 2014 - Fecha aceptado: agosto II de 2014

** Epidemiólogo clínico, Universidad El Bosque. Bogotá DC, Colombia.

* Epidemiólogo clínico, Universidad El Bosque. Residente III de Neurología.

Fundación Universitaria de Ciencias de la Salud. Bogotá DC, Colombia. 


\section{Introduc ción}

Las infecciones del SNC son una causa muy común de morbimortalidad y con el uso indiscriminado de antibióticos han aparecido nuevos agentes etiológicos, con cambio en la clínica y aumento en la resistencia bacteriana a los antibióticos convencionales. Es fundamental que tanto el neurólogo clínico como el médico general conozcan las condiciones y las características de nuestra población, para evitar retrasos en el diagnóstico y secuelas neurológicas irreversibles. Los estudios observacionales son de carácter demográfico, lo que permite que sean apropiados para establecer metas orientadas a una determinada acción. Pretendemos generar nuevas hipótesis y una línea de investigación, basados en datos de la población que se investiga. ${ }^{1}$

La meningitis es la inflamación de las meninges producida por diversas causas, dentro de las cuales se encuentran las infecciosas que pueden producirse por bacterias, virus, hongos o protozoarios. Es la más común de las infecciones del SNC y corresponde a la invasión de la leptomeninge (píamadre y aracnoides) por vía hematógena, por contigüidad como en las sinusitis y otitis, por invasión directa como en el trauma durante procedimientos quirúrgicos. ${ }^{2}$

\section{Epidemiología}

La incidencia anual de MB en EEUU es de 2 a 10 casos por 100.000 habitantes, es más frecuente en el paciente pediátrico y las tasas de morbimortalidad en diferentes series varía del 25 a $60 \% .^{3}$

\section{Microorganismos}

Con el uso de vacunas contra Haemophilus influenzae se ha originado un cambio en la epidemiología. En la actualidad el orden de frecuencia es $S$. pneumoniae (47\%), N. meningitidis (25\%), estreptococos del grupo B $(12 \%)$ y L. monocyotogenes $(8 \%)$, esta última de predominio en adultos mayores. ${ }^{4} \mathrm{El} S$. pneumoniae es la causa más frecuente de MB en adultos entre 19 y 60 años. ${ }^{5}$

\section{Clínica}

El curso clínico puede ser insidioso en el 90\% de los casos o fulminante en el 10\%. Entre los síntomas y signos se encuentran fiebre, cefalea, rigidez de nuca, alteración de la conciencia y convulsiones. ${ }^{6}$ La triada clásica (fiebre, cefalea y rigidez de nuca) solo se observa en $40 \%$ de los adultos jóvenes y $90 \%$ presenta alguno de los dos síntomas. Las convulsiones son más frecuentes en los niños, hasta una tercera parte de estos las presentará y en ellos los síntomas pueden ser inespecíficos, observando letargo, astenia, fiebre y eritema. $^{7}$

\section{Diagnóstico}

El análisis de LCR es fundamental para el diagnóstico y tratamiento. En general las MB producen pleocitosis de predominio polimorfonuclear neutrófilo, con disminución de la glucosa y aumento de las proteínas. ${ }^{8} \mathrm{La}$ inmunoelectroforesis de LCR, la aglutinación de látex y los estudios de coagulación pueden detectar antígenos bacterianos comunes en 70 a $100 \%$ de los casos. El cultivo requiere cerca de 48 horas y es positivo en $80 \%$ de los casos de MB. ${ }^{9}$ En el $90 \%$ la pleocitosis es alta y con frecuencia hay más de 100 leucocitos por campo. ${ }^{10}$

Los estudios neurorradiológicos en la fase inicial la TAC puede ser normal, pero con frecuencia se puede observar realce de las meninges. ${ }^{11}$ La imagen por resonancia magnética está indicada en casos complicados con síndromes convulsivos o signos focales, ya que estos estudios tienen más sensibilidad para detectar áreas focales de meningoencefalitis o vasculitis en la secuencia de FLAIR. ${ }^{12,13}$

\section{Tratamiento}

La elección del antibiótico depende de los factores de riesgo, pero la mayor parte de los regímenes incluye cefalosporinas de tercera o cuarta generación. ${ }^{14}$

La ampicilina se debe utilizar ante la sospecha de L. monocytogenes (adultos mayores, VIH, recién 
nacidos). ${ }^{15}$ Meropenem es una alternativa para las cefalosporinas de tercera y cuarta generación ${ }^{16} \mathrm{La}$ vancomicina es una buena elección pero teniendo en cuenta que su acción en el LCR depende de la inflamación en las meninges. ${ }^{17}$

\section{Materiales y métodos}

Estudio observacional descriptivo tipo serie de casos. El tiempo “0” fue determinado por el diagnóstico clínico de infección del SNC (es de notificación obligatoria). Se identificaron todos los pacientes con la clasificación internacional CIE-10, por el sistema génesis a nivel país. Se revisaron un total de 304 historias clínicas con diagnóstico de meningitis. De estas, 42 cumplieron los criterios de inclusión. Población elegible: pacientes con diagnóstico de meningitis infecciosa en el Hospital Central de la Policía en el período 2004-2010.

Criterios de inclusión: aquellos con diagnóstico probable de meningitis infecciosa, que cumplieron con los criterios clínicos y paraclínicos. Criterios de exclusión: historias clínicas mal diligenciadas, datos insuficientes, diagnóstico errado, diferente y paraclínicos incompletos. Selección de la muestra: muestreo por conveniencia.

\section{Resultados}

Se revisaron 304 historias con diagnóstico potencial de meningitis. Se reclutaron 42 de pacientes con meningitis que cumplían los criterios de inclusión clínicos y paraclínicos entre los años 2004 y 2010. Hombres $24(57,1 \%)$ y mujeres $18(42,9 \%)$ (Figura 1). La edad promedio fue 30,44 años (D.E. $+27,39$ ) con un rango entre 0 y 80 años. La distribución geográfica mostró 28 procedentes de Bogotá (66,7\%). Los meses en los cuales hubo mayor número de consultas fueron en orden descendente: octubre (siete, 16\%), noviembre (seis, $14,3 \%$ ) y mayo (cinco 11,9\%). En estos tres meses se efectuaron dieciocho consultas equivalentes al $42,9 \%$ del total, mostrando una estacionalidad que no coincide con los meses tradicionales de lluvia. Con respecto a los años de consulta se aprecia que la mayoría de los casos fueron diagnosticados en 2004 (once
26,2\%), seguido por 2007 ocho 19,0\%) y 2005 (siete 16,7\%) (Figura 2).

En cuanto a las comorbilidades, las dividimos en generales, agudas y del SNC siendo más frecuentes las dos últimas (Tabla 1). En nuestra población, solo dos pacientes eran VIH positivos. Dentro de los síntomas más importantes aparece la fiebre en $33(78,6 \%)$, seguida por cefalea en 23 (54,8\%), vómito en 13 (31\%) y cambios en el comportamiento 11 (26,2\%). Otros síntomas como escalofrío, malestar general y enfermedad diarreica aguda, ocurrieron en 15 pacientes $(53,7 \%)$ y solo seis de los 42 presentaron convulsiones (14,3\%).

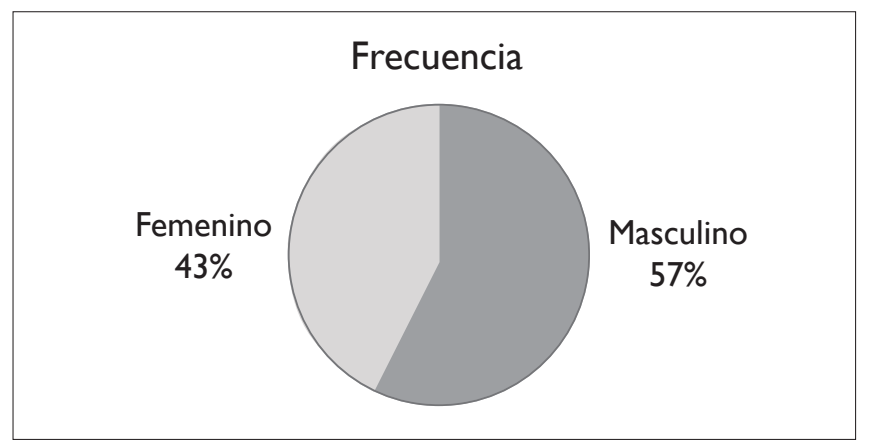

Figura I. Distribución por género.

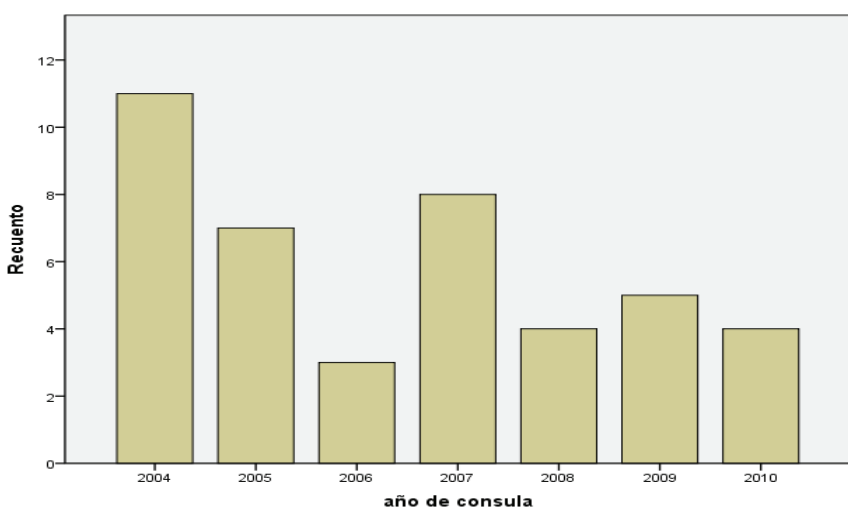

Figura 2. Frecuencia de consultas por año.

\begin{tabular}{|l|c|c|}
\hline \multicolumn{3}{|c|}{ Tabla I. Comorbilidades } \\
\hline Comorbilidades en general & $30(71.4 \%)$ & NO \\
\hline Comorbilidades agudas & $17(40.5)$ & $25(59.5 \%)$ \\
\hline Comorbilidades en SNC & $13(31 \%$ & $29(69 \%)$ \\
\hline
\end{tabular}


Importante destacar que de los 42 con diagnóstico de meningitis, $20(47,6 \%)$ no presentaban signos meníngeos al ingreso. Entre los 22 que tenían irritación meníngea, 19 mostraron rigidez nucal (86\%), $21(50 \%)$ déficit neurológico y 10 tenían Glasgow menor de 15 .

En cuanto a los resultados de la punción lumbar y los estudios solicitados en LCR, se encontró que este apareció turbio en 31 pacientes $(73,8 \%)$. En el recuento de leucocitos en LCR la media fue de 779,33/ $\mu \mathrm{L}$ (DE: + $790 / \mu \mathrm{L})$ y el rango entre 3 y $2250 / \mu \mathrm{L}$. En 30 muestras se presentó predominio neutrofílico y en seis linfocítico. En cuanto a proteínas en LCR la media fue de $188,05 \mathrm{mg} / \mathrm{dL}(\mathrm{DE}:+157,3 \mathrm{mg} / \mathrm{dL}$ ) y el rango entre 1 y $621 \mathrm{mg} / \mathrm{dL}$. La lactatodeshidrogenasa (LDH) tuvo una media de 211,42 U/mL (DE: + 396,9 U/mL) y el rango entre 4 y $1766 \mathrm{U} / \mathrm{mL}$. La glucorraquia media de $37,47 \mathrm{mg} / \mathrm{dL}$ (DE: + 48,9 mg/dL) y el rango entre 0 y $299 \mathrm{mg} / \mathrm{dL}$. El látex para criptococo en LCR se realizó en tres muestras de las cuales una fue positiva. Se practicó gram en 30 y en 8 de 14 (33,3\%) fueron cocos gram positivos. El cultivo de LCR fue positivo en 16 muestras $(38,1 \%)$ (Tabla 2). La adenosindeaminasa (ADA) se practicó en cuatro pacientes y una fue positiva iniciándose manejo para TBC (26 mg/dL).

\begin{tabular}{|c|c|}
\hline Tipo de microorganismo & Frecuencia (\%) \\
\hline Cultivos negativos & $19(54,29 \%)$ \\
\hline Neisseria meningitides & I $(2,86 \%)$ \\
\hline Staphylococcus warneri & I $(2,86 \%)$ \\
\hline Streptococcus pneumonia & $2(5,71 \%)$ \\
\hline Staphylococcus aureus & I $(2,86 \%)$ \\
\hline Staphylococcus epidermidis & $2(5,71 \%)$ \\
\hline Chryseobacterium meningosepticum & I $(2,86 \%)$ \\
\hline Staphylococcus hominis & I (2,86\%) \\
\hline Streptococcus sanguinis & I $(2,86 \%)$ \\
\hline Streptococcus agalactiae & I $(2,86 \%)$ \\
\hline Listeria monocitogenes & $2(5,7 \mid \%)$ \\
\hline Streptococcus alphahemolyticus & I $(2,86 \%)$ \\
\hline Enterococcus faecalis & I $(2,86 \%)$ \\
\hline Micrococcus luteus & I $(2,86 \%)$ \\
\hline Streptococcus oralis & I (2,86\%) \\
\hline
\end{tabular}

En cuanto a pruebas sanguíneas el hemograma reveló un promedio de $12.740 /$ leucocitos $/ \mathrm{mL}$ con un rango entre 1.000 y $32.000 / \mathrm{mL}$.

Se practicaron 34 tomografías simples de cráneo de las cuales $22(52,4 \%)$ mostraron anormalidades, seis con cambios secundarios a patologías neuroquirúrgicas, cuatro con edema cerebral difuso, de estos dos no demostraron en la resonancia magnética cerebral cambios sugestivos de meningitis aguda (Tabla 3).

El manejo de su patología infecciosa aguda en la fase inicial fue: 41 recibieron antibióticos $(97,6 \%)$, cinco antivirales $(11,9 \%)$ y antimicóticos cuatro $(9,5 \%)$. De los 42 pacientes 33 recibieron tratamiento antibiótico combinado, siendo el más utilizado ceftriaxona más vancomicina (Tabla 4). Un porcentaje importante, 16 de 42 pacientes, requirieron unidad de cuidado intensivo $(38,1 \%)$, falleciendo $5(31,3 \%)$ (Tabla 5).

\begin{tabular}{|c|c|}
\hline Resultado & Frecuencia (5\%) \\
\hline Normal & $12(28,6)$ \\
\hline Anormal & $22(52,4)$ \\
\hline Sin imágenes & $8(19,0)$ \\
\hline Total & $42(100)$ \\
\hline
\end{tabular}

\begin{tabular}{|l|c|}
\hline \multicolumn{2}{|c|}{ Tabla 4. Antibióticos prescritos } \\
\hline \multicolumn{1}{|c|}{ Antibióticos prescritos } & $\begin{array}{c}\text { Número de } \\
\text { pacientes }\end{array}$ \\
\hline Ceftriaxona-vancomicina & 9 \\
\hline Ceftriaxona & 5 \\
\hline Ceftriaxona-ampicilina & 3 \\
\hline Ceftriaxona- ampicilina sulbactam & 3 \\
\hline Otros & 21 \\
\hline
\end{tabular}

\begin{tabular}{|l|c|}
\hline \multicolumn{2}{|c|}{ Tabla 5. Pacientes que requirieron $\mathrm{UCl}$} \\
\hline \multicolumn{1}{|c|}{ Pacientes } & Número de pacientes \\
\hline Pacientes requirieron UCl & $16(38.1 \%)$ \\
\hline Pacientes fallecidos en $\mathrm{UCl}$ & $5(31.3 \%)$ \\
\hline Pacientes recuperados en $\mathrm{UCl}$ & $1 \mathrm{I}(68.8 \%)$ \\
\hline Total de pacientes & $42(100 \%)$ \\
\hline
\end{tabular}

\section{Pacientes que ingresaron a UCI}

Se describen los casos que entraron a UCI para observar el comportamiento tan agresivo de las infecciones del SNC, que tuvieron cultivo positivo. 
Caso 1: recién nacida en postoperatorio de ventriculostomía por hidrocefalia con irritabilidad, fiebre, leucocitosis de $21.000 / \mathrm{mL}$ con neutrofilia, LCR turbio, hiperproteinorraquia e hipoglucorraquia. Cultivo positivo para Staphylococcus epidermidis manejado con vancomicina y cefotaxime. Caso 2: recién nacida con status epilepticus y signos meníngeos, LCR turbio, hiperproteinorraquia, hipoglucorraquia, cultivo positivo para Chryseobacterium meningosepticum; tratada con ceftriaxona y ampicilina. Caso 3: niña de 9 años con antecedente de masa carótida intracavernosa, ingresó por fiebre, cefalea y vómito, signos meníngeos positivos, LCR turbio, glucorraquia y proteinorraquia normales, cultivo positivo para Streptococcus sanguinis manejada con ceftriaxona y vancomicina. Caso 4: niño de un mes de edad con antecedentes de hipogamaglobulinemia; ingresó por fiebre y crisis convulsiva, LCR turbio, hiperproteinorraquia y glucorraquia indetectable. Cultivo positivo para Streptococcus agalactiae, tratado con ampicilina, ceftriaxona y cefepime. Caso 5: paciente de 54 años con cefalea, vómito y fiebre, signos meníngeos positivos, Glasgow 14/15, hiperproteinorraquia, hipoglucorraquia, cultivo positivo para listeria monocitogenes, manejada con rifampicina, fluconazol y ceftriaxona. Caso 6: recién nacido con fiebre e irritabilidad, incremento de leucocitos en LCR e hipoglucorraquia. Cultivo positivo para Staphylococcus epidermidis, manejado con ampicilina y cefepime. Llama la atención que fueron pacientes en los extremos de la vida con, factores de riesgo importantes e infecciones por gérmenes atípicos.

\section{Pacientes que fallecieron}

Siete de los 42 pacientes murieron durante el tiempo en que padecieron meningitis $(16,7 \%)$.

Caso 1: niño de año y medio en postoperatorio por un meduloblastoma, con neutropenia y en quimioterapia. Presentó fiebre, vómito, irritabilidad, hipoglucorraquia y LDH en $1.776 \mathrm{U} / \mathrm{mL}$. El gram de LCR mostró cocos gram positivos, no tuvimos acceso al cultivo y los antibióticos usados fueron cefepime y vancomicina. Caso 2: mujer de 51 años, con antecedentes de neumonía aspirativa y edema cerebral. Al ingreso fiebre, escalofrío, estupor y rigidez nucal. Glasgow 3/15. Manejo con ampicilina, sulbactam y ceftriaxona. No hubo cultivo. Caso 3: mujer de 23 años con antecedente de VIH y meningitis crónica ingresó con cefalea y fiebre, alteración del estado de consciencia y pancitopenia. Se manejó con ceftriaxona, claritromicina, trimetoprim, anfotericina y clindamicina. Caso 4: hombre de 20 años con cefalea, fiebre, vómito, convulsión, rigidez nucal, pupilas mióticas al ingreso, LCR turbio, linfocitosis, hiperproteinorraquia, hipoglucorraquia y cultivo negativo. Se manejó con aciclovir, ceftriaxona y vancomicina. Caso 5: niño de 6 meses con antecedente de parto pretérmino, bajo peso al nacer. Ingresó con fiebre, vómito, fontanelas abombadas, LCR turbio, neutrofilia, hiperproteinorraquia y glucorraquia no detectada. Cultivo positivo para Streptococcus pneumoniae. Tratamiento con ceftriaxona y oxacilina. Caso 6: mujer de 60 años con antecedente de neumonía basal derecha e insuficiencia renal crónica, ingresó por fiebre, tos, vértigo, nistagmos horizontal, Glasgow 14/15, LCR con leve hiperproteinorraquia e hipoglucorraquia. Cultivo positivo para Staphylococcus hominis, tratamiento con vancomicina y ampicilina-sulbactam.

Caso 7: hombre de 67 años, con antecedente de EPOC, que cursó con disartria, cefalea, fiebre, convulsión, signos meníngeos, hemiparesia derecha y LCR con hiperproteinorraquia e hipoglucorraquia. Cultivo negativo. Tratado con ceftriaxona y vancomicina.

\section{Disc usión}

En la presente serie de 42 pacientes predomina el género masculino, encontramos pacientes desde recién nacidos hasta ancianos, la mayoría procedentes de Bogotá, debido a que el hospital de la policía es centro de referencia. El 42,9\% de las consultas se efectuaron durante los meses de octubre, noviembre y mayo. Se aprecia una tendencia a la disminución en el número de casos, con un pico en el año 2007. De las 304 historias clínicas con diagnóstico potencial de meningitis, 42 cumplieron con los criterios de inclusión.

El $71 \%$ de los pacientes tuvieron comorbilidades, $31 \%$ asociadas con el sistema nervioso central, dos fueron VIH positivos y en uno se aisló un germen atípico. Los síntomas más frecuentes fueron inespecíficos (cefalea, fiebre y vómito), lo cual puede confundirse con otras 
patologías dificultando el diagnóstico. Seis pacientes $(14,3 \%)$, presentaron convulsiones y veinte $(47,6 \%)$ no cursaron con signos meníngeos al ingreso. Entre los $22(86 \%)$ que tuvieron signos meníngeos, 19 (86\%) mostraron rigidez nucal. La mitad de los pacientes desarrollaron algún déficit neurológico.

El LCR fue turbio en 31 pacientes $(73,8 \%)$ con valores medios de leucocitos $779,33 / \mu \mathrm{L}$, de proteinoorraquia $188,05 \mathrm{mg} / \mathrm{dL}$ y de glucorraquia $37,47 \mathrm{mg} / \mathrm{dL}$. Sigue siendo esta la herramienta principal para el diagnóstico, los que estuvieron en UCI y los que fallecieron tenían cambios importantes relacionados con la severidad de la patología.

Dentro de las neuroimágenes predominó el uso de la TAC simple, de las cuales el 52,4\% fue anormal, sin mostrar las características típicas que se describen en la literatura médica. El tratamiento antibiótico combinado más usado fue ceftriaxona y vancomicina sin que haya unidad de criterio en el inicio de la antibióticoterapia implementada en los pacientes por lo bizarro de los síntomas iniciales; 38,2\% necesitaron UCI y cinco fallecieron; otros dos murieron sin haber requerido UCI, cifra importante para una patología no tan frecuente, Fallecieron más los pacientes en extremos de la vida, con múltiples comorbilidades y que requirieron manejo en UCI. Seis de estos fallecidos tenían factores de riesgo importantes para mortalidad por meningitis.

En los microorganismos aislados no se aprecia una frecuencia importante de alguno de ellos. Estos fueron: Streptococcus pneumoniae, Staphylococcus epidermidis y Listeria monocitogenes, cada uno con dos casos y un porcentaje de $5,71 \%$. Las bacterias tradicionales como el meningococo no son tan comunes, lo cual nos sugiere que los gérmenes etiológicos están cambiando y la mortalidad estuvo asociada con gérmenes no tan frecuentes y con comorbilidades múltiples.

El presente estudio nos invita a pensar en meningitis como diagnóstico diferencial, vemos que sigue siendo una patología con una alta morbimortalidad y es obligatoria pensarla cuando nos encontremos frente a un paciente con síntomas como cefalea, fiebre y vómito. Sugerimos realizar estudios observacionales analíticos para identificar factores de riesgo asociados con mortalidad por meningitis infecciosa.

\section{Referencias}

1. Medina MI, Sánchez Pérez E, Daza Barriga JS, Yepes Sanz M, Duque Samper A, Roncallo del Portillo A, et al. Enfermedades infecciosas del Sistema Nervioso Central. Toro Gómez J, Yepes Sanz M, Palacios Sánchez E. Neurología. 2a ed. Bogotá: Manual Moderno; 2010. p. 255-316

2. Roos KL, Tunkel AR, van de Beek D, Sheld WM. Acute Bacterial meningitis. In: Scheld WM, Whitley RJ, Durack DT, editors. Infections of the central nervous system. 3rd ed. Philadelphia: Lippincott William Wilkins; 2004. p. 365-419.

3. Tebruegge M, Curtis N. Epidemiology, etiology, pathogenesis, and diagnosis of recurrent bacterial meningitis. Clin Microbiol Rev. 2008 Jul; 21(3):519-37.

4. Tique V, Alvis N, Parodi R, Bustos A, Mattar S. Meningitis agudas en Córdoba, Colombia 2002-2004. Rev. salud pública. 2006; 8 (Sup.1): 33-46.

5. Grupo GREBO. Información resistencia bacteriana 2007-2008. Bogotá: Grebo; 2009

6. Molina SI, Pérez AR, García M. Meningitis bacteriana: estudio georreferencial en ciudad de La Habana durante 1998. Rev Cubana Med Trop. 2001; 53: 204-11.

7. de Oliveira Rossoni AM, Dalla Costa LM, Bonato Berto D, Santos Farah S, Gelain M, de Cunto Brandileone, MC, et al. Acute bacterial meningitis caused by Streptococcus pneumoniae resistant to the antimicrobian agents and their serotypes. Arq Neuro-Psiquiatr. 2008; 66(3-A): 509-15.

8. Attia J, Hatala R, Cook DJ, Wong JG. Does this adult patient have acute meningitis? JAMA. 1999; 282(2):175-81

9. Schut ES, Westendorp WF, de Gans J, Kruyt ND, Spanjaard L, Reitsma JB, et al. Hyperglycemia in bacterial meningitis: a prospective cohort study. BMC Infect Dis. 2009, 9:57.

10. Dzupova O, Rozsypal H, Prochazka B, Benes J. Acute bacterial meningitis in adults: predictors of outcome. Scand J Infect Dis. 2009; 41(5):348-54

11. Restrepo Jaramillo BN, Aguirre C, Upegui G, Carrizosa J. Características del manejo hospitalario y oportunidad de la consulta en pacientes con meningitis por Neisseriameningitidis y Haemophilusinfluenzae tipo B. Medellín, Colombia. CES Med. 2005; 19(2):47-55.

12. Castañeda Orjuela C, Alvis Guzmán N, de la Hoz Restrepo F. Impacto de la enfermedad por Streptococcus pneumoniae en población adulta mayor en Bogotá, Colombia, 2008. Rev. salud pública. 2010; 12(1): 38-50

13. Agudelo CI, Sanabria OM, Ovalle MV. Serogroup Y meningococcal disease, Colombia. Emerg Infect Dis. 2008 Jun; 14(6):990-1.

14. Rubinsky RO. Streptococcus pneumoniae: epidemiología y resistencia antimicrobianos de las enfermedades invasoras en Latinoamérica. Rev Chil Infect. (2001); 18 (Supl. 1): 10-14

15. de Carlos D, Jordá R, Hidalgo O, Salas A. Tratamiento de las meningitis agudas bacterianas [monografía en Internet]. Islas Baleares: El Comprimido; 2002. [citado 2014 mayo 20]. Disponible en: http://www.elcomprimido.com/FARHSD/ PROTMENINGITIS.pdf

16. American Academy of Pediatrics. Committee on Infectious Diseases, Canadian Paediatric Society. Infectious Diseases and Immunization Committee. Meningococcal disease prevention and control strategies for practice-based physicians. Pediatrics. 1996; 97:404-12.

17. Honda H, Warren DK. Central nervous system infections: meningitis and brain abscess. Infect Dis Clin N Am. 2009; 23(3): 609-23. 